Regards sur l'économie allemande

Bulletin économique du CIRAC

$103 \mid 2011$

Varia

\title{
L'apprentissage en entreprise au cœur de la compétitivité allemande
}

René Lasserre

\section{(2) OpenEdition}

\section{Journals}

Édition électronique

URL : http://journals.openedition.org/rea/4354

DOI : 10.4000/rea.4354

ISBN : 978-2-8218-1136-2

ISSN : 1965-0787

\section{Éditeur}

CIRAC

\section{Édition imprimée}

Date de publication : 16 décembre 2011

Pagination : 13-18

ISSN : 1156-8992

\section{Référence électronique}

René Lasserre, «L'apprentissage en entreprise au cœur de la compétitivité allemande », Regards sur l'économie allemande [En ligne], 103 | décembre 2011, mis en ligne le 01 décembre 2013, consulté le 20 avril 2019. URL : http://journals.openedition.org/rea/4354 ; DOI : 10.4000/rea.4354 


\section{L'apprentissage en entreprise au cœur de la compétitivité allemande}

\section{René Lasserre}

On se livre avec assiduité, depuis plusieurs mois, à l'exercice de la comparaison des facteurs de compétitivité France-Allemagne. Et on passe au crible tous les indicateurs statistiques officiels pour tenter d'identifier la ou les formules permettant de découvrir le secret des performances allemandes. Mais à force de ne vouloir considérer que des facteurs chiffrables dont l'objectivité semble hors de tout soupçon, puisque les données ainsi obtenues ont été scientifiquement calculées, on en parvient à croire que ces facteurs-coûts permettraient à eux seuls d'expliquer les différences dans le dynamisme de nos deux économies. Et de fait, on en oublie l'essentiel: le rôle de ces facteurs hors coût ou autres "soft skills" sans lesquels les facteurs coûts ne peuvent ni s'expliquer ni se comprendre, puisqu'ils sont soustraits à leur contexte national respectif qui seul leur confère un sens.

Parmi ces facteurs hors coût qui éclairent la compétitivité allemande, il en est un dont on ne mesure pas toutes les implications : c'est l'apprentissage dans le système de formation duale. On l'identifie essentiellement comme l'explication du faible taux de chômage des jeunes en Allemagne. Or s'il assure effectivement la fluidité de la transition formation/emploi, il joue aussi un rôle clé dans le haut niveau de qualification de la population allemande et sa hausse continue. Car au savoir théorique et technique des salariés ainsi formés s'ajoute une " compétence d'action " qui leur confère la capacité à s'adapter à un environnement et des process en mutation, et d'innover. C'est là le 'secret' de la compétitivité et de la force d'innovation des entreprises allemandes, et par-dessus tout du Mittelstand industriel qui forme un grand nombre de jeunes. C'est là aussi, de ce fait, le 'secret' d'une culture entrepreneuriale au fondement de l'identité sociale allemande sur laquelle repose sa compétitivité économique.

\section{La voie royale de la formation professionnelle dans tous les grands secteurs de l'économie}

En Allemagne, l'apprentissage constitue en quelque sorte la voie royale de la formation professionnelle initiale. Celle-ci est en effet majoritairement organisée sous la forme de l'apprentissage, que l'on désigne généralement sous le terme générique de "système dual », puisqu'il se déroule au moins sur deux lieux de formation : l'entreprise formatrice comme pivot et centre de gravité du système, et l'école professionnelle (publique) où sont dispensés, à raison d'environ $20 \%$ du temps, des enseignements généraux et techniques théoriques.

En dehors des formations de l'enseignement supérieur, menant à des fonctions d'encadrement, le système dual est ainsi la forme dominante d'acquisition des qualifications professionnelles pour tous les emplois d'opérateurs qualifiés et de responsables techniques supérieurs. II accueille chaque année plus de la moitié d'une classe d'âge, soit 1,6 million d'apprentis, et ce dans tous les secteurs d'activité, principalement l'industrie, la finance et le commerce $(60 \%$ des nouveaux contrats en 2010), l'artisanat (27\%), les professions libérales (environ $8 \%$ ), les $5 \%$ restants se répartissant entre les formations duales dans les ser-

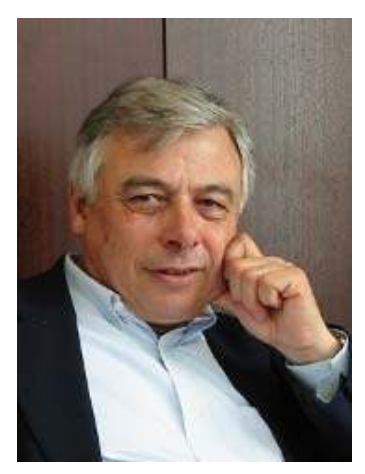

Prof. René Lasserre, Directeur du CIRAC
Le système dual concerne...

... plus de la moitié d'une classe d'âge 
Le socle d'une population active hautement qualifiée

La responsabilité des entreprises..

... assure un pilotage de l'offre de formation par le marché vices publics, l'agriculture, l'économie domestique et la marine marchande. A côté des formations par apprentissage, la formation professionnelle initiale fait également appel à des formations en milieu scolaire pour certaines formations spécifiques (professions paramédicales ou artistiques) et aussi, de façon croissante, à des formations pré-professionnelles permettant à des jeunes en difficulté ou ne disposant pas d'un bagage suffisant d'accéder ultérieurement à l'apprentissage.

Le système dual est par ailleurs le principal agent pourvoyeur et multiplicateur de qualification professionnelle de l'économie allemande: sur les 38,7 millions de personnes actives occupées en 2008, 59\% disposaient d'un diplôme de formation professionnelle initiale achevée, dont la plupart l'ont acquise dans le système dual (Schmidt, 2010). Parmi ces quelque 23 millions de détenteurs d'un diplôme professionnel, un peu plus de 10 \% disposent en outre d'une qualification reconnue de formateur par apprentissage (technicien/ contremaître/ maître-artisan). Ces diplômes de formateurs relèvent de la formation professionnelle continue, et leur obtention suppose la réussite préalable d'une formation professionnelle initiale (duale en règle générale) et une expérience professionnelle de plusieurs années dans le métier appris, de sorte que le système dual lui-même génère pour l'essentiel la continuité du potentiel de formation. $\mathrm{A}$ côté de ce socle de formation professionnelle et technique très large, allant de l'ouvrier professionnel qualifié (Facharbeiter) au technicien ou à l'employé supérieur et intégrant environ $15 \%$ de bacheliers, près de $27 \%$ des actifs sont titulaires d'un diplôme d'enseignement supérieur. La part de la population active employée ne disposant pas d'une formation professionnelle initiale achevée n'a cessé de se réduire au cours des deux dernières décennies et se situe en dessous des $15 \%$, soit moitié moins que la moyenne des pays de l'OCDE qui tourne autour de $30 \%$.

\section{Caractéristiques et principes organiques du système}

Concrètement, le système dual représente donc actuellement quelque 1,6 million d'apprentis par an, répartis sur trois années de formation en général, soit 560000 entrées en formation (chiffres de 2009/10). Ces jeunes sont formés à l'un des 348 métiers codifiés par le système dual. Outre sa première caractéristique qui est la pluralité des lieux et niveaux d'apprentissage, le fonctionnement du système repose sur trois grands principes organiques : la responsabilité des entreprises, l'implication des partenaires sociaux et la référence à un métier dans la construction et la transmission de la qualification. C'est la conjonction de ces trois éléments qui assure l'efficacité du système et en fait un facteur de compétitivité coût et hors coût déterminant de l'entreprise allemande.

Par tradition, fondée à la fois sur l'expérience historique et sur la loi, les entreprises sont reconnues, tant par l'Etat que par le corps social, comme l'opérateur naturel et principal de la formation professionnelle initiale et en sont le principal maître d'œuvre, puisque c'est à elles que revient l'initiative de s'engager à dispenser une formation aux apprentis qu'elles recrutent et de mettre en œuvre les moyens nécessaires pour la mener à bien.

Les entreprises ne sont cependant pas obligées de former : une entreprise sur quatre, parmi les quelque 3,5 millions enregistrées en Allemagne (dont plus de $99 \%$ de PME), s'engage dans la formation professionnelle duale. Conformément au principe de subsidiarité, les pouvoirs publics, quant à eux, se contentent de fixer le cadre légal pour les entreprises sur la base de la définition d'un certain nombre de requis minima, tant pour ce qui est du contenu de la formation que de l'organisation matérielle de celle-ci.

Cette liberté d'initiative et cette autonomie de gestion reconnues à l'entreprise assurent un pilotage par le marché et une régulation par les besoins, tant pour ce qui est de l'offre quantitative de formation que de l'évolution des qualifica- 
tions. Dès lors qu'elle décide de s'investir dans la formation, l'entreprise s'engage dans un investissement conséquent qui l'oblige à évaluer au mieux ses besoins de recrutement à moyen terme en même temps qu'à offrir des contenus de formation qui répondent le mieux possible aux besoins de qualification qu'induisent l'évolution des techniques, des méthodes de production et des marchés.

L'expérience prouve que les entreprises sont généralement les mieux placées pour anticiper les besoins d'emploi et de compétences de leur branche d'activité, et que dès lors qu'elles s'engagent dans cette démarche, elles s'efforcent d'investir en conséquence, et vont généralement très largement au-delà des minima fixés par le règlement de formation défini pour chaque profession. En ce sens, elles sont les meilleurs garants possibles d'une modernisation continue de la formation professionnelle. En outre, l'implication des entreprises allemandes, y compris des entreprises moyennes, dans la pratique formatrice, et la capacité de gestion et de développement des compétences qui en découle, constituent à grande échelle le ferment privilégié où se nourrit leur dynamique d'innovation.

A côté des organisations patronales représentant les entreprises, les syndicats sont étroitement associés à la régulation du système et y font valoir les intérêts des salariés et des apprentis. Ils sont systématiquement consultés dans la définition des contenus et des conditions matérielles de réalisation de la formation, et ce à tous les niveaux auxquels des décisions relatives à la formation professionnelle sont prises : le niveau fédéral interprofessionnel, le niveau fédéral de la branche professionnelle, le niveau régional interprofessionnel et professionnel, le niveau de l'entreprise où la formation est organisée et où le Conseil d'entreprise dispose d'un droit de co-décision.

L'objectif de cette concertation est de chercher l'adhésion des partenaires sociaux, et tout particulièrement celle des salariés, à chaque niveau de décision, de façon à faire prévaloir sur le terrain la logique de la gestion concertée sur l'intervention réglementaire des pouvoirs publics. Seuls sont codifiés les grands principes de base faisant consensus. Les pouvoirs publics ne jouent que le rôle de médiateur, avec un pouvoir d'arbitrage existant en droit, mais rarement utilisé dans la réalité. Celui-ci s'opposerait en effet à la tradition d'une gestion contractuelle de la formation professionnelle, tant au niveau de la branche qu'à celui de l'entreprise, ainsi qu'au principe de subsidiarité qui privilégie et valorise la responsabilité des acteurs, réservant aux pouvoirs publics une position quelque peu en retrait et un rôle d'animateur-promoteur plutôt que de pilote ou d'arbitre.

La formation, dont les contours et les contenus sont essentiellement déterminés par la négociation entre les partenaires sociaux, donne lieu à l'attribution d'un diplôme qui fait référence à un métier. Celui-ci se définit comme un ensemble structuré et cohérent de connaissances et de compétences techniques acquises dans un champ professionnel donné, reconnu au niveau national et légitimé collectivement par tous les acteurs socioprofessionnels d'une même branche. Le métier est un élément constitutif du système dual auquel les partenaires sociaux, notamment les syndicats, restent très fortement attachés dans leurs efforts de modernisation. On lui attribue plusieurs fonctions essentielles dont celles-ci : le métier est facteur d'identité individuelle, en ce sens qu'il offre à l'individu des perspectives claires d'épanouissement en termes de carrière, de mobilité et de revenu, mais également facteur d'identité collective, en ce sens qu'il assure une forte lisibilité des compétences acquises sur un marché du travail par ailleurs très segmenté.

C'est au niveau de la branche professionnelle que l'architecture des métiers et la structuration des qualifications s'organisent et que le marché du travail se régule, aussi bien en termes de recrutement que de rémunération et de promotion. C'est en particulier au niveau de la convention de branche que sont définis
La forte implication des partenaires sociaux...

\section{... assure la \\ légitimité consensuelle} du système

Formation dans un métier : gage de reconnaissance professionnelle et sociale

Métier et régulation de branche structurent la gestion de l'emploi et le rapport salarial 
le volume et la structure de l'offre de places d'apprentissage et que sont fixés les niveaux de rémunération des apprentis. De ce fait, la participation des partenaires sociaux à la définition des conditions-cadre de la politique de formation professionnelle globale est un enjeu essentiel qui trouve ensuite son prolongement effectif dans la politique conventionnelle de branche, en particulier en matière de gestion contractuelle des qualifications. Par cette continuité dans l'implication des acteurs économiques est ainsi assuré, aux différents niveaux où ils exercent leur responsabilité décisionnelle, un lien organique entre la politique de formation et de qualification professionnelles des différents métiers, la régulation du marché du travail, la rémunération et la promotion des compétences.

\section{Un rôle essentiel dans la valorisation du capital humain}

Rôle clé dans la compétitivité hors coût

Compétence d'action

Fluidité de la transition formation-emploi

Un taux de chômage des jeunes parmi les plus bas d'Europe
La formation professionnelle duale contribue de façon fondamentale à préserver, voire à conforter, par son effort de rationalisation permanent, aussi bien la valorisation des compétences que la capacité d'innovation, et par voie de conséquence, la compétitivité de l'économie allemande. C'est notamment le lien quasi-organique entre système de formation et système d'emploi qui permet aux entreprises d'assurer une qualification en adéquation avec leurs besoins à moyen terme. En effet, la formation duale préfigure très largement à l'année $\mathrm{N}-3 / \mathrm{N}-5$ la structure des emplois et les possibilités de promotion, notamment dans l'industrie manufacturière où le Facharbeiter (ouvrier qualifié ayant achevé une formation duale) est devenu dans le dernier quart du $20^{\mathrm{e}}$ siècle la figure emblématique de la compétitivité industrielle allemande sur les marchés mondiaux.

Cette formation ne signifie pas seulement une acquisition de connaissances techniques théoriques par le jeune, mais de par l'importance de la place de l'entreprise comme lieu de formation, ce dernier est en mesure d'acquérir tant un savoir-faire qu'un savoir-être professionnels dans des conditions réelles de production. Le jeune ainsi formé dispose donc d'une Handlungskompetenz, d'une " compétence d'action », qui le distingue par rapport aux jeunes issus d'une formation professionnelle essentiellement scolaire.

La forte implication des partenaires sociaux comme instances de conceptualisation des contenus et de contrôle à tous les niveaux décisionnels garantit une formation technique de très haut niveau, mais également ouverte et générale, de sorte que les compétences professionnelles et surtout sociales de base acquises puissent être appliquées à d'autres contextes institutionnels que celui de l'entreprise d'accueil. C'est à ce titre qu'elle est reconnue au niveau national par l'ensemble des professionnels d'une branche. Dans le cas de figure le plus fréquent, le système dual permet ainsi aux jeunes une qualification de qualité et leur assure une insertion professionnelle sans rupture ni frictions à la fin de leur formation.

En effet, le taux d'embauche global des apprentis à l'issue de leur formation est d'environ $60 \%$, avec cependant de fortes disparités selon les secteurs d'activité, la région, les aléas de la conjoncture économique générale ou sectorielle, voire l'origine sociale et le sexe du jeune ainsi formé. Toujours est-il que la formation duale permet à une part importante de la jeunesse non seulement l'obtention d'une qualification de haut niveau, mais également un accès précoce (à 20 ou 21ans) et une transition plutôt satisfaisante vers un emploi stable et généralement hautement productif. Elle favorise ainsi l'autonomie de ces jeunes et leur participation pleine et entière à la vie économique et sociale sans passer par des périodes plus ou moins longues d'activité peu qualifiée ou faiblement qualifiante, voire de précarité et de chômage. Le taux de chômage des jeunes de moins de 25 ans se situe en effet en 2011 à $9,2 \%$ des actifs de cette classe d'âge, ne dépassant que d'un peu plus de deux points le taux de chômage moyen de l'ensemble des actifs qui s'établit à $7 \%$. Les jeunes Allemands se 
classent ainsi, depuis plusieurs décennies, dans le haut du classement européen pour l'insertion professionnelle.

Au plan macro-économique, la formation professionnelle en entreprise est un facteur essentiel de valorisation du potentiel de la main-d'œuvre jeune. Elle lui assure en effet une insertion optimisée au moment où elle est à la fois la plus dynamique et la mieux adaptable et où elle vient d'acquérir les connaissances techniques les plus récentes. Elle est aussi, il convient de le noter, par un taux d'activité précoce et élevé, un facteur positif dans le financement des systèmes de protection sociale.

$\mathrm{Au}$ plan micro-économique, elle est certes un investissement relativement coûteux à court terme, que l'on peut chiffrer en moyenne de l'ordre de $5 \%$ à $6 \%$ de la masse salariale en flux initial constant, mais qui s'amortit voire se neutralise intégralement au fil des deux à trois années d'apprentissage grâce à la prestation productive croissante de l'apprenti. Mais cet investissement initial devient fortement et durablement positif dès l'embauche, d'autant que les apprentis nouvellement recrutés font généralement preuve d'une bonne stabilité dans leur premier emploi (de l'ordre de 5 ans dans les principales branches) pour s'assurer un parcours professionnel à la fois durable et valorisant. Sous l'effet conjugué d'une productivité optimale, d'une rémunération modérée de début de carrière, sans parler de considérables et incertaines dépenses de recrutement-formation dont il permet de se dispenser pour assurer la relève et la continuité du potentiel d'emplois du cœur de métier, l'investissement-formation initial est un levier de rentabilité et d'efficacité incontestable de l'entreprise allemande. Bien qu'il soit très difficile à chiffrer précisément, on peut à notre sens l'estimer en terme d'avantage net ex-post de coût salarial au moins à l'équivalent de l'effort initial.

A L'HEURE OÙ LE BENCHMARK des coûts de production et des facteurs de compétitivité hors-prix avec notre principal concurrent et partenaire est à l'ordre du jour de ce côté-ci du Rhin, il apparaît ainsi que l'apprentissage en entreprise assure aux entreprises allemandes un avantage compétitif tout à fait significatif, que ce soit en termes d'avantages sectoriels ou macro-économiques indirects ou en matière de coûts salariaux directs, sans parler d'efficacité managériale dans la gestion et la valorisation des ressources humaines.

En tant que producteur de qualifications et de savoir-faire, l'apprentissage apparaît enfin comme un atout essentiel dans le processus d'innovation. Loin de se limiter à l'acquisition d'un savoir technique dans un domaine spécialisé (comme on l'interprète souvent à tort vu de France), et parce qu'il est au contraire est en prise directe avec l'évolution des techniques et des processus de production, l'apprentissage à l'allemande développe parmi les opérateurs un potentiel diversifié de compétences-clés d'analyse, d'autonomie et de contrôle. En cela il est à la charnière du processus d'innovation : il constitue à la fois le tissu de l'innovation permanente et incrémentale de process qui est le point fort des entreprises allemandes, en même temps que le terrain d'expérimentation, de mise en œuvre et de diffusion de techniques nouvelles au stade de la production. En ce sens, l'apprentissage est bien au cœur de la chaîne de valeur et de la compétitivité hors prix qui, sur les marchés des biens à haute valeur ajoutée, permet aux entreprises allemandes de faire la différence.

\section{Indications bibliographiques}

- « Bildung und Wissenschaft », in DeStatIS, Statistisches Jahrbuch 2011, Wiesbaden, 2011

-BUNDESINSTITUT FüR BeRUfSBILdUNG, Datenreport zum Berufsbildungsbericht 2011. Informationen und Analysen zur Entwicklung der beruflichen Bildung, consultables sur www.bibb.de/ veroeffentlichungen
Levier de rentabilité et d'efficacité pour l'entreprise 
-BUNDESMINISTERIUM FÜR ARBEIT UND SOZIALES, Arbeitskräftereport, novembre 2011, consultable sur www.bmas.de/SharedDocs/Downloads/DE/PDF-Publikationen/a859_arbeitskraeftereport. pdf;jsessionid=ACF313ABBAF2D489D5C0C7AF060D0D29?_blob=publicationFile

-BUNDESMINISTERIUM FÜR BILDUNG UND FORSCHUNG, Berufsbildungsbericht 2011, consultable sur www.bmbf.de

-Deutsche Telekom Stiftung, Bundesverband der Deutschen Industrie (eds), Innovationsindikator 2011, consultable sur www.innovationsindikator.de

- «Fachkräftenachwuchs », Dossier, Magazin für Soziales und Familie, n ${ }^{\circ}$, 12/2011, consultable sur www.bundesregierung.de/Content/DE/Magazine/01MagazinSozialesFamilie/12/12.html

- LASSERRE R., "L'apprentissage: un vecteur de compétitivité ", Echanges, La revue des dirigeants financiers, $\mathrm{n}^{\circ} 289$, juillet-août 2011 (Dossier : Allemagne : mais comment-font-ils ?)

- Lasserre R., Zettelmeier W., Etude du système de formation professionnelle en Allemagne, réalisée pour la Cour des Comptes ( $5^{e}$ chambre), 2007 (non publiée).

- LasserRe R., LATTARd A., La formation professionnelle en Allemagne, Editions du CIRAC, Paris, 1993,

-Schmidt N., "Auswirkungen des Strukturwandels der Wirtschaft auf den Bildungsgrad der Bevölkerung ", Wirtschaft und Statistik, $n^{\circ} 6 / 2010$

-Wingerter C., «Der Eintritt junger Menschen in das Erwerbsleben. Ergebnisse der Arbeitskräfteerhebung 2009 », Wirtschaft und Statistik, février 2011

-ZetTelmeier W., "Allemagne : la transition formation/éducation/emploi », Regards sur l'économie allemande, $\mathrm{n}^{\circ} 75$, mars 2006. 